\title{
- 㩆-
}

La gestión de las cuentas a cobrar y a pagar: el factoring y el confirming

CARlos JaVIER SANZ SANTOLARIA

ESCUELA UNIVERSITARIA DE ESTUDIOS SOCIALES UNIVERSIDAD DE ZARAGOZA

Resumen: En el marco del actual proceso de globalización económica, las sociedades procuran optimizar sus recursos financieros por razones de competitividad y de beneficio. En este artículo se exponen de una forma clara y sencilla el régimen contable y económico, así como las principales cuestiones relativas a dos figuras de financiación modernas, el factoring y el confirming.

Palabras clave: Financiación, deudores, acreedores.

Managing accounts receivable and payable: factoring and conforming

Abstract: Within the current process of economic globalisation, societies try to optimize their financial resources in order to maximize their competitiveness and profits. This paper explains in a clear, simple man- 
ner, the economic and accounting organisation of two new financial practices, i.e. factoring and confirming, in addition to other important matters related to such practices.

Keywords: Financing, debtors, creditors.

\section{La gestión de las cuentas \\ a cobrar y a pagar: \\ el factoring \\ y el confirming}

\section{Aspectos generales}

Los deudores de la actividad o deudores por operaciones de tráfico son activos de la empresa que como tales representan beneficios económicos futuros, expresando el derecho de la empresa a exigir un compromiso u obligación adquiridos por terceros normalmente como consecuencia de créditos concedidos.

Esos beneficios económicos futuros pueden consistir en la conversión de los derechos en efectivo u otros medios líquidos equivalentes o en el derecho a percibir bienes o servicios en el futuro e incluso a reducir pagos en el futuro.

Como el resto de activos, estos deben ser registrados en el balance de situación, y en función de si su vencimiento supera o no el ciclo de explotación, pueden ser tanto activos circulantes o corrientes como no corrientes.

La NIC 39 incluye en las cuentas a cobrar originadas por la actividad a aquellos activos financieros que proceden de la actividad ordinaria de la empresa y que es de suponer que se mantendrán hasta su cobro en el momento del vencimiento. Son activos creados por la empresa a cambio de proporcionar efectivo, bienes o servicios directamente al deudor.

Siguiendo los criterios fijados por la NIC 39, la valoración inicial de las cuentas a cobrar debe hacerse por su valor razonable, teniendo en cuenta además los costes de transacción atribuibles a la operación.

Por su parte, los acreedores de la actividad son elementos de pasivo que representan obligaciones de la empresa a terce- 
ros como consecuencia de su actividad habitual, por lo que también son denominados acreedores por operaciones de tráfico. Expresan el crédito concedido por otras entidades o personas e implican el compromiso de la empresa de hacer frente a las mismas entregando efectivo $u$ otros activos financieros, o en ocasiones, bienes y servicios.

Como cuentas de pasivo que son, estas deben estar en el pasivo del balance de situación, y en función de si su vencimiento es, o no, superior al ciclo de explotación, pueden ser tanto de pasivo circulante o corriente como no corriente.

Las cuentas a pagar representan obligaciones de pago originadas en la empresa como consecuencia del aplazamiento en la adquisición de bienes y servicios que la empresa utiliza en el desarrollo de su actividad. De acuerdo con las normas internacionales, estas cuentas a pagar son pasivos financieros, cumpliendo, por tanto, los requisitos incluidos en la definición de pasivo financiero: un compromiso que supone una obligación contractual de entregar efectivo u otro activo financiero a otra empresa, o de intercambiar instrumentos financieros con otra empresa, bajo condiciones que son potencialmente desfavorables.

En el marco del actual proceso de globalización económica las sociedades procurar optimizar sus recursos financieros por razones de competitividad y de beneficio. Así, en los últimos años las entidades financieras han desarrollado dos nuevos productos financieros cuya utilización alcanza dos vertientes importantes, de un lado, el cobro de las ventas (el factoring), y de otro, la disponibilidad de los recursos financieros y el pago de las deudas (el confirming). Ambas operaciones pueden entenderse como las dos caras de una misma moneda. En el factoring es el proveedor el que acude a la entidad financiera para que le gestiones el cobro de las facturas emitidas al cliente. Por el contrario, en el confirming es el deudor el que acude a la entidad financiera para que le gestione el pago de las facturas emitidas contra él por el proveedor.

En ambos casos se trata de contratos financieros de naturaleza atípica, lo que supone que, aunque se encuentran plenamente reconocidos por la realidad social y son de frecuente utilización en el tráfico jurídico y mercantil, carecen de regulación legal específica, aún cuando la ley contenga alusiones a ellos.

\section{El factoring}

Puede definirse como el contrato por el que un empresario cede la titularidad de los créditos comerciales que ostenta frente a sus clientes a otro empresario especializado (entidad de factoring), prestando este último una serie de servicios relacionados con los créditos que se ceden.

La entidad de factoring, en ocasiones, asume el riesgo de insolvencia del deudor cedido, lo que da lugar a la distinción de dos tipos de factoring: el factoring sin recurso, en el que el cedente responde de la existencia y legitimidad del crédito pero no de la solvencia del deudor cedido; y el factoring con recurso, que es aquél en el que el cedente responde de la existencia y legitimidad del crédito y de la solvencia del deudor cedido, por lo que en caso de insolvencia del deudor cedido la entidad de factoring puede repetir contra su cliente, el cedente.

En otras ocasiones, se pacta entre cedente y entidad de factoring que esta última entregue al cedente un anticipo, esto es, una parte del nominal de los créditos cedidos y cuyo cobro ha de gestionar la entidad de factoring, antes de que se produzca el cobro efectivo. En estos casos existe una financiación por parte de la entidad de factoring al cedente.

Existen otra serie de servicios que puede prestar la entidad de factoring relacionados con el contrato de factoring, como son la realización de informes de solvencia, estudios de mercado, etc.

En contraprestación a los servicios que presta la entidad de factoring, el cedente satisface a la entidad de factoring una remuneración, consistente normalmente en un porcentaje sobre los créditos cedidos, pudiendo además recibir la entidad de factoring el pago de un interés en el caso de que ésta anticipe al cedente el pago de los créditos antes de su cobro efectivo.

Es posible encontrar diversos tipos de factoring en función de varios criterios de clasificación:

- En función del riesgo que corra la entidad de factoring, tal y como se ha expuesto anteriormente, el factoring puede ser con recurso o sin recurso.

- Según el momento de nacimiento de los créditos que se ceden existen cesiones de créditos presentes y de créditos futuros. 
- En función de la naturaleza del deudor, el factoring podrá ser de tres tipos. Cuando el deudor sea una Administración Pública Central, Autonómica o Local el factoring podrá ser factoring sector público. Cuando el deudor sea del sector privado se reconocen dos tipos de factoring: factoring sector privado nacional, y cuando el deudor se halle ubicado fuera del territorio nacional, factoring sector privado no nacional o de exportación.

- En función del momento de pago de los créditos por la entidad de factoring existe factoring con pago al cobro, en el que la entidad de factoring para al cedente una vez que ha cobrado el crédito del deudor; factoring con pago al cobro pero con una fecha límite, en el que la entidad de factoring, sin recurso, paga al cedente en el momento del cobro o en una fecha prefijada posterior en el caso de no haber conseguido el cobro; y el factoring con pago a una fecha fija, independientemente de que la entidad de factoring haya cobrado o no el crédito.

Las principales ventajas del factoring son:

- El cedente ya no tiene que destinar medios materiales y humanos para gestionar el cobro de los créditos, lo que supone una reducción de tiempo y de costes en seguimiento de cobros, impagados, comunicaciones, burocracia asesoramiento jurídico, contenciosos, etc.

- En el caso de que se haya pactado que la entidad de factoring pague al cedente los créditos antes de su vencimiento, ello permite al cedente obtener liquidez. Se obtiene financiación automática de sus ventas paralelamente con su crecimiento.

- Cuando se trate de factoring de exportación, se elimina la incertidumbre a la hora de plantearse la expansión internacional. El exportador, además de estar protegido contra posibles insolvencias del deudor, se beneficia de una gestión exterior de cobro especializada, ya que generalmente la realiza una entidad del propio país del deudor.

- En el factoring sin recursos el cedente elimina el riesgo de insolvencia de los deudores. Además, la empresa reduce su cuenta de clientes a cobrar, lo que permite mejorar su sol- vencia. En general, permite, con independencia de su estructura financiera, desenvolverse y crecer con seguridad.

Por su parte, los inconvenientes del factoring son:

- El factoring puede crear una cierta confusión entre los deudores a la hora de realizar los pagos, si no se ha comunicado la cesión de los créditos a un nuevo acreedor o cesionario.

- En caso de cesión de créditos futuros, el cedente ha de imponer a los futuros deudores el pago de sus créditos al nuevo acreedor.

Sin lugar a dudas, la principal característica del factoring es la no existencia del derecho de regreso sobre los créditos que han sido transferidos. Esto hace que en el contrato de factoring se pacten cuidadosamente las condiciones que deben reunir los créditos sometidos al mismo.

La empresa de factoring (denominada "factor") investiga la solvencia de los futuros clientes, los cuales deberán ser expresamente aceptados por ésta. Se ocupa del cobro, incluso de morosos o insolventes. El hecho de que el "factor" asuma la posible insolvencia del deudor significa que la empresa usuario del factoring satisfará, en el coste de la operación, no solamente la comisión por la administración y gestión del cobro, sino también el "riesgo de insolvencia" que calculará técnicamente el factor. El coste de la operación se verá incrementado por los intereses que correspondan al anticipo de fondos, en el caso de que éste haya sido solicitado.

Se suele establecer un anticipo máximo de fondos, el que corresponda al $80 \%$ o $90 \%$ del valor nominal de las facturas, quedando retenido el resto como garantía de la empresa de factoring. La retención será liquidada cuando termine la operación.

El coste total de las operaciones sometidas al contrato de factoring está en función de la solvencia de los compradores, frecuencia de la facturación, importe medio de las facturas, período medio de vencimiento y zona geográfica fundamentalmente. Como es fácil deducir, debido a la transferencia del riesgo de insolvencia, así como del resto de los servicios que presta, el coste de la "factorización" de los créditos de la empresa es más oneroso que el descuento bancario. Además 
de las ventajas e inconvenientes antes citados, el mayor coste, junto con la desvinculación de la empresa-vendedora de los problemas de pago del cliente-comprador, hacen que no sea esta una modalidad muy extendida. No obstante, pueden señalarse algunas características ventajosas, entre las que destaca la seguridad en la realización de las cuentas a cobrar y la sencillez en la administración de las mismas.

En nuestro Plan General de Contabilidad no hay previstas cuentas específicas para este tipo de actividad, pero la contabilidad de la empresa que decide utilizar el factoring se podría desarrollar creando las siguientes subcuentas de clientes:

\section{CLIENTES, FACTURAS ENVIADAS A LA}

APROBACIÓN DEL FACTOR (430)

$$
\text { a CLIENTES (430) }
$$

Por el importe de las facturas enviadas a la empresa de factoring CLIENTES, FACTURAS APROBADAS POR EL FACTOR (430)

(por las facturas que han sido aprobadas)

CLIENTES (430)

por las facturas que han sido rechazadas)

\section{a CLIENTES, FACTURAS ENVIADAS}

A LA APROBACIÓN DEL FACTOR (430)

Por el abono que realiza el factor de las facturas aprobadas, bien en el momento del cobro, bien anticipadamente si se ha solicitado, se hará:

TESORERÍA (57)

INTERESES POR DESCUENTO DE FACTURAS (664)

SERVICIOS DE PROFESIONALES INDEPENDIENTES.

COMISIONES DE FACTORING (623)

CLIENTES, RETENCIÓN DEL FACTOR (430)

\section{a CLIENTES, FACTURAS APROBADAS} POR EL FACTOR (430)

Por último, señalar respecto a la información a incluir en los estados financieros, que en línea con la información exigida por la NIC 32 y NIC 39 para cada clase de activo y pasivo financiero, los estados financieros deben recoger, entre otra, la siguiente información relativa a las cuentas a cobrar y a las cuentas a pagar:
- Su naturaleza, alcance y las políticas y métodos contables utilizados.

- Información sobre su valor razonable, riesgos de tipo de interés, de crédito y de liquidez.

- Efectos en el estado de resultados y en patrimonio y correcciones de valor.

- Incumplimiento de contratos y pagos vencidos no atendidos.

\section{El confirming}

Dentro de los distintos productos financieros el confirming aparece como nuevo medio de pago que permite simultáneamente conceder una serie de servicios al deudor y ser un medio de financiación y garantía para el acreedor.

Se define como la oferta de unos servicios financieros por parte de una entidad especializada, que generalmente es una compañía de factoring. Estos servicios son ofrecidos por la compañía a un usuario, a fin de que éste los pueda utilizar como medio de pago y cancelación de las deudas contraídas con sus proveedores. La entidad financiera se constituye así, en gestora de los pagos que su cliente le ordena realizar para liquidar sus compras, en uso del correspondiente contrato. En cumplimiento de esa orden, y al vencimiento de la operación, la entidad financiera emite un cheque o transferencia por cuenta del cliente, que anuncia previamente y remite a su destinatario, cancelando así la deuda existente. El confirming es, pues, un producto o medio de pago que garantiza al acreedor la percepción de la deuda. Esta garantía contempla el pleno reconocimiento del pago, y que el mismo se realizará en el término de tiempo acordado y será abonado por una entidad financiera.

El contrato de confirming es atípico, careciendo de regulación en nuestro Derecho, si bien presenta notas características de varios contratos en función de las prestaciones que se acuerden con la entidad de confirming:

- Puede considerarse una comisión mercantil para el pago de las deudas, en la que la entidad actúa como intermediaria.

- También puede considerarse un arrendamiento de servicios. 
- En caso de que la entidad financie a su cliente, podría hablarse de apertura de crédito en cuenta corriente o de préstamo bancario.

- Por último, si anticipa los pagos a los acreedores encontraríamos elementos del contrato de descuento.

En consecuencia, al contrato de confirming le serán aplicables las normas correspondientes reguladoras de dichas figuras, de carácter civil y mercantil.

A diferencia del factoring, en el que existe cesión de créditos, en el caso del confirming no existe transmisión de las deudas a la entidad de confirming, pues ésta se encarga exclusivamente de la gestión de su pago, sin que exista asunción de deudas. Por lo tanto, el deudor sigue siendo el mismo, sólo que es la entidad de confirming la que se encarga del pago de las deudas de su cliente.

La posición de dicha entidad se encuadra, en principio, en la figura del pago por tercero, ya que según el Código Civil, cualquier persona hacer el pago por cuenta de otro, tenga o no interés en el cumplimiento de la obligación.

El confirming siempre contiene un servicio de gestión de pago, ya que la entidad financiera paga al proveedor en nombre del cliente, pero esta gestión de pago admite distintas modalidades, tanto desde el punto de vista del cliente como desde la posición del proveedor.

Así, en función de los servicios que la entidad de confirming se comprometa a prestar al cliente deudor, podemos encontrar:

- Un confirming simple, en el que la entidad simplemente gestiona los pagos del deudor, haciendo de intermediaria.

- El confirming de inversión, en el que el cliente deudor anticipa a la entidad de confirming el pago de las facturas, que posteriormente ésta ha de abonar a los proveedores, y el cliente se deduce de la cantidad que ha de pagar a la entidad de confirming el descuento financiero correspondiente.

- El confirming de financiación, en el que el cliente deudor retrasa el pago de las facturas hasta una fecha posterior a la de su vencimiento, con lo que la entidad de confirming le concede una financiación por la que le cobra, además de la comisión pactada, los intereses correspondientes al tiempo que media entre la fecha del vencimiento y la del pago efectivo.
Por otra parte, y desde el punto de vista del proveedor, también se puede distinguir entre:

- Confirming sin descuento, en el que el proveedor ha de dirigirse a la entidad de confirming en la fecha del vencimiento para el cobro, sin que pueda percibirlo anticipadamente, pero sin que tenga que hacer nada ni abonar cantidad alguna a la entidad. En este caso es el proveedor el que asume el riesgo de impago del cliente, a no ser que la entidad haya acordado paralelamente financiar a este último.

- Confirming con descuento, que siempre es "sin recurso", en el que el proveedor cobra su crédito de la entidad de confirming anticipadamente respecto a la fecha de su vencimiento, debiendo satisfacer un interés en concepto de descuento, y en el que en el momento del vencimiento de la factura la entidad únicamente puede dirigirse a su cliente para el cobro, sin que pueda solicitar al proveedor la devolución del anticipo en el caso de que el cliente no pague.

- Por último, el proveedor también podría, si así se acuerda con la entidad de confirming, retrasar el cobro de las facturas más allá de su fecha de vencimiento, en cuyo caso recibiría una remuneración al tipo de interés pactado.

Respecto de las ventajas e inconvenientes del confirming, se pueden señalar:

- Para el cliente deudor.

El confirming le permite reducir el coste del departamento de pagos, al homogeneizarse el sistema de pago y al eliminarse las tareas de gestión. Asimismo, incrementa su poder de negociación frente a los proveedores y le permite incrementar el plazo de pago sin tener que negociar individualmente con cada proveedor, posibilitándole asimismo el retrasar el pago a los proveedores en el caso del confirming con financiación. Sin embargo, el hecho de no pagar el empresario obligado directamente puede generar confusión y desconfianza en los acreedores; y, además, ha de adecuarse a las condiciones de la entidad de confirming tanto a nivel de condiciones de las operaciones como en lo referente a las directrices señaladas por la entidad. 
- Para la entidad de confirming.

Esta figura le permite ampliar su gama de servicios y captar nuevos clientes (proveedores) para otras de sus líneas de negocio. Sin embargo, la rentabilidad de estas operaciones suele ser reducida, y existe la posibilidad de riesgos imprevistos.

- Para el proveedor.

El confirming le permite reducir los costes relacionados con los cobros, así como obtener los cobros anticipadamente mediante el descuento, a la vez que incrementa la seguridad de los cobros y permite al proveedor domiciliar los pagos en la entidad financiera, con un incremento de las relaciones entre proveedor y entidad financiera. Entre los inconvenientes se encuentra el que normalmente las condiciones de descuento no son negociables y que se ha de dirigir necesariamente a la entidad de confirming elegida por el empresario deudor.

En resumen, ambos contratos, con sus inherentes particularidades, el factoring es un sistema de gestión de cobros y el confirming un sistema de gestión de pagos, comparten importantes similitudes. Así, en el confirming pueden confluir ambas figuras cuando se conceda al proveedor la posibilidad de que se le anticipe el pago mediante la cesión de sus créditos, a modo y semejanza del factoring.

De forma esquemática, ambas fórmulas pueden representarse de la forma siguiente:

\section{ESQUEMA DEL FACTORING}

\section{Venden mercancías} con pago aplazado

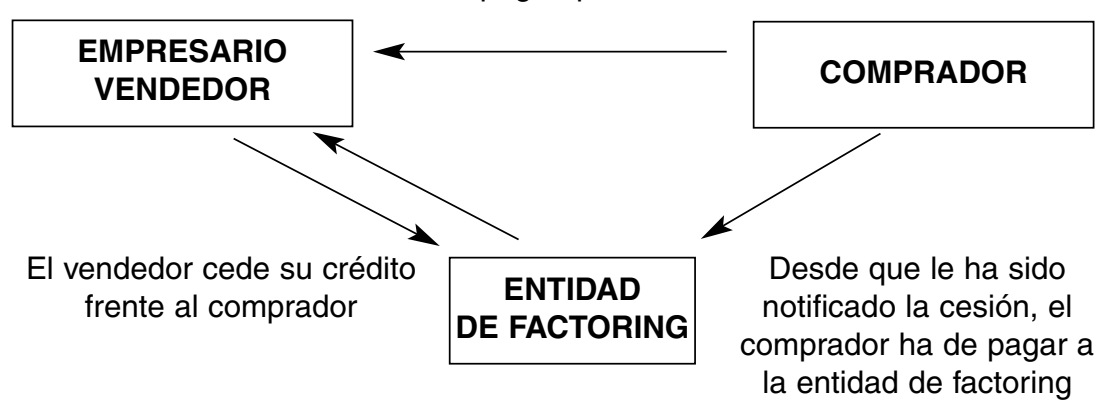

ESQUEMA DEL FACTORING

Venden mercancías

con pago aplazado

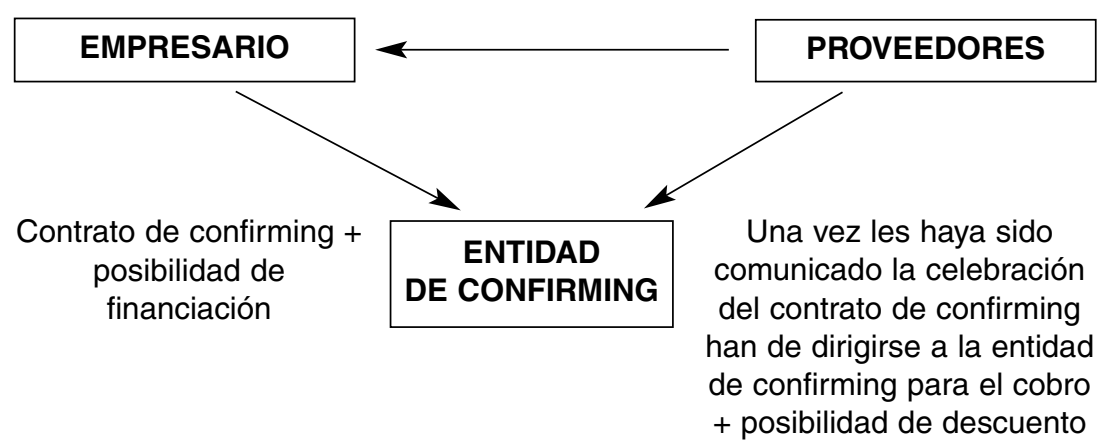

\section{Bibliografía}

AMAT, O. (coordinador) (2003): Comprender las Normas Internacionales de Contabilidad. Barcelona: Gestión 2000.

ASOCIACION ESPAÑOLA DE CONTABILIDAD Y ADMINISTRACION DE EMPRESAS (AECA): Documentos sobre Principios Contables. Madrid: AECA.

BRUSCA, I. et al. (2004): Introducción a la contabilidad financiera: un enfoque internacional. Barcelona: Ariel.

CEA, J.L. (2002): Armonización contable internacional y reforma de la contabilidad en España. Madrid: ICAC.

GINER INCHAUSTI, B. (2003): El nuevo proceso de regulación contable en España: cambios en el proceso y en las normas. Madrid: Revista AECA, $\mathrm{n}^{\circ}$ 65, pp. 13-16.

GONZALO ANGULO, J.A. (2003): Las NIIF: contabilidad y control. La cara oculta de las normas internacionales. Madrid: Revista AECA, $n^{\circ} 65$, pp. 3-12.

INSTITUTO DE CONTABILIDAD Y AUDITORIA DE CUENTAS (2002): Informe sobre la situación actual de la contabilidad en España y líneas básicas para abordar su reforma: Libro Blanco para la reforma de la contabilidad en España. Madrid: ICAC. 
INTERNATIONAL ACCOUNTING STANDARDS BOARD (IASB) (2003a): International Accounting Standard 32. Financial Instruments: Disclosure and Presentation. Londres: IASCF.

INTERNATIONAL ACCOUNTING STANDARDS BOARD (IASB) (2003b): International Accounting Standard 39. Financial Instruments: Recognition and Measurement. Londres: IASCF.

TUA, J. (coordinador) (2002): Marco conceptual para la información financiera. Análisis y comentarios. Madrid: AECA.

VELA, M.; MONTESINOS, V. y SERRA, V. (2000): Manual de Contabilidad. Barcelona: Ariel Economía.

YEBRA, O. (director) (2002): Normas Internacionales de Contabilidad 2002-2003. Desarrollo y comentarios. Madrid: Francis Lefebre. 